\title{
Geometric constraints in the growth of nanotube-templated polymer monolayers
}

\author{
J. N. Coleman and M. S. Ferreira ${ }^{\text {a) }}$ \\ Physics Department, Trinity College Dublin, Dublin 2, Ireland
}

(Received 19 September 2003; accepted 1 December 2003)

\begin{abstract}
We present a simple and general model that describes the ordered assembly of polymer strands on nanotube surfaces. Energetically favorable coiling angles are identified based on geometric constraints that limit the maximum coverage of polymers on the walls of the nanotubes. The coiling angles can be controlled by selecting the appropriate nanotube diameters, opening the possibility of engineering the strength of composite fibers. The model is applied to two different polymeric molecules; namely, polyacetylene and a polyphenylenevinylene derivative. (C) 2004 American Institute of Physics. [DOI: 10.1063/1.1643538]
\end{abstract}

More than a decade after the discovery of carbon nanotubes, these nanoscale cylindrical structures are still the subject of intensive scientific research due to their fascinating physical properties. Electronic and mechanical properties of nanotubes have been the main focus of study and have resulted in a number of applications, such as the construction of single wall nanotube (SWNT)-based transistors ${ }^{1}$ and electromechanical devices, ${ }^{2}$ to name but a few. However, it is widely believed that the most immediately accessible applications are those involving the reinforcement of plastics using carbon nanotubes.

Significant progress has already been made toward this goal. It has been shown that Young's modulus of polyvinyl alcohol (PVA) can be increased by a factor of 2 with loading levels of less than $1 \%$ multiwalled nanotubes. ${ }^{3}$ This high level of reinforcement was attributed to the formation of a crystalline polymer coating at the nanotube surface leading to enhanced polymer-nanotube interfacial stress transfer. Studies using similar nanotubes and a noncrystalline polymer resulted in much lower levels of reinforcement strongly suggesting that a well-ordered polymer coating is vital for effective stress transfer. ${ }^{3}$ In addition, PVA-SWNT fibers have been produced with strength and toughness superior to steel and Kevlar, respectively. ${ }^{4}$ As PVA is known to crystallize on the surface of a SWNT, ${ }^{5}$ it is thought that an ordered interface is crucial to the astounding mechanical properties of these materials.

Since the crystallinity of the polymer coating seems to play such a fundamental role in the strength of composite fibers, it is worth investigating the conditions for the ordered interaction of long one-dimensional-like molecules around carbon nanotubes. Experimental observations at low coverage show that the polymers tend to coil at well defined angles to the nanotube axis. ${ }^{6}$ It has been suggested that such a high degree of regularity in the polymer-nanotube alignment reflects the major role played by the chirality of the underlying hexagonal lattice in selecting the preferential coiling angle of the polymer. ${ }^{7}$ However, in this letter, we question this assumption by showing that energetically favor-

${ }^{a)}$ Electronic mail: ferreirm@tcd.ie able coiling angles can be identified even when the polymernanotube interaction does not resolve the atomic structure of the underlying lattice. In this case, purely geometric factors introduce constraints on the maximum coverage of polymers and limit the energy gain involved in the process. It is important to stress that we are not questioning the generally accepted view that the crystalline order of thin films is crucially dependent on the atomic structure of the underlying substrate. However, concerning the ordered coating around a narrow cylindrical structure like a carbon nanotube, the role played by the geometrical constraints may outweigh the lattice-resolved contribution from the underlying hexagonal structure.

This can be explained by a simple model that calculates the amount of energy required to wrap polymer strands around a nanotube. We assume two energetically competing factors contributing to the alignment process: The van der Waals-based binding energy between the parts and the elastic-energy cost required to bend the polymer. We impose the former to be position independent, which results from taking the average potential over the surrounding atoms. Rather than resolving the atomic structure of the underlying lattice, taking the average binding energy is justified by the fact that the molecular structure of most polymers cannot be commensurate with the nanotube lattice without introducing a significant amount of strain. In other words, the energy is not necessarily minimized by a direct mapping onto the underlying lattice.

Concerning the second contribution to the energy balance, we model the polymer as a semi-flexible continuous stripe of width $\omega$ so that the energy cost of bending the polymer can be obtained by simply calculating the elastic energy involved in wrapping the stripe at an angle to the tube axis. It is worth clarifying that the effective width of the polymer strands is defined here as the lateral separation between nearest-neighbor polymers in the crystalline phase. Based on this model, we are able to predict the total energy and probability density as a function of coiling angle for arbitrary polymer-nanotube combinations. Two particular cases are considered here, namely polyacetylene (PA) and a polyphenylenevinylene derivative, PmPV.

We start by considering one infinitely long polymer 

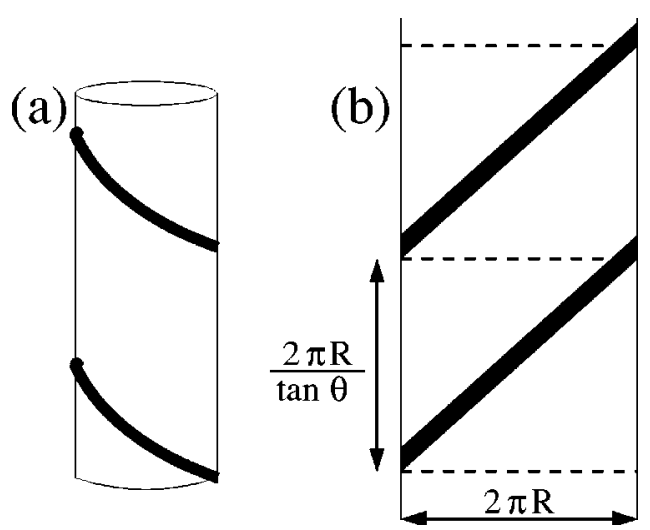

FIG. 1. (a) Schematic representation of a single polymer strand coiling along the axis of a nanotube. (b) In the two-dimensional depiction, the unwrapped tube is represented by an infinitely long stripe of width $2 \pi R$ and the coiling angle $\theta$ defines a unit cell of length $2 \pi R / \tan \theta$. The black stripe of width $\omega$ symbolizes the polymer strand.

coiled around an equally long nanotube of radius $r$, as schematically shown in Fig. 1. The polymer lies on a cylindrical surface of radius $R=r+\delta$, where $\delta$ is the van der Waals distance to the tube surface, hereafter considered to be constant $(\delta=3.5 \AA)$. The binding energy per unit length of polymer is angle independent and given by $B=-\sigma \omega$, where $\sigma$ is a measure of the average binding energy per unit polymer area. The published values of $\omega$ are $0.48 \mathrm{~nm}$ for $\mathrm{PA}^{8}$ and $2.1 \mathrm{~nm}$ for $\mathrm{PmPV}^{9}$ while values of $\sigma$ can be deduced to be $378 \mathrm{meV} / \mathrm{nm}^{2}$ and $770 \mathrm{meV} / \mathrm{nm}^{2}$ for $\mathrm{PA}^{8,10}$ and $\mathrm{PmPV}$, respectively. ${ }^{9}$ This results in values of $B$ of $181 \mathrm{meV} / \mathrm{nm}$ for $\mathrm{PA}$ and $1617 \mathrm{meV} / \mathrm{nm}$ for PmPV.

Concerning the elastic energy cost, we call it the coiling energy $C$ defined as $C=D \kappa^{2},{ }^{11}$ where $D$ is the stiffness constant of the polymer and $\kappa=\sin ^{2} \theta / R$ is the respective curvature of its helicoidal path. The stiffness constant is in turn related to the so-called persistence length $L_{p}$ of the polymer through $D=L_{p} k_{b} T,{ }^{11} k_{b} T$ being the thermal energy. The persistence length has been reported as $1.3 \mathrm{~nm}$ for $\mathrm{PA}^{12}$ and approximately $10 \mathrm{~nm}$ for PPV derivatives. ${ }^{13}$ By writing in units of $D / R^{2}$, it is evident that the coiling energy is larger for stiffer polymers and smaller nanotube diameters. For a single strand, we can then write the total energy per unit length of polymer as

$$
E_{1}(\theta)=B+C=-\sigma \omega+\frac{D \sin ^{4} \theta}{R^{2}} .
$$

Maximum values of $C$ for PA and PmPV wrapping a nanotube of diameter $1.3 \mathrm{~nm}$ are $33 \mathrm{meV} / \mathrm{nm}$ and $250 \mathrm{meV} / \mathrm{nm}$, respectively. This result is significant as it shows that the coiling energy tends to be small in comparison to the binding energy. This surprising result is due to the fact that stiffer polymers tend to have a larger width resulting in a greater binding energy per unit length. Therefore, for low coverage, no clear preferential angles can be identified since the angleindependent binding energy seems to dominate the process.

To model the crystalline phase of the polymer, we now consider the situation of many strands wrapping around the nanotube with a fixed coiling angle. In this case, the energy is scaled with the number of polymers. It is evident from Fig. 1(b) that for a given angle $\theta$, the maximum number of strands covering the nanotube is geometrically constrained to Downloaded 19 Mar 2009 to 134.226.1.229. Redistribution subject
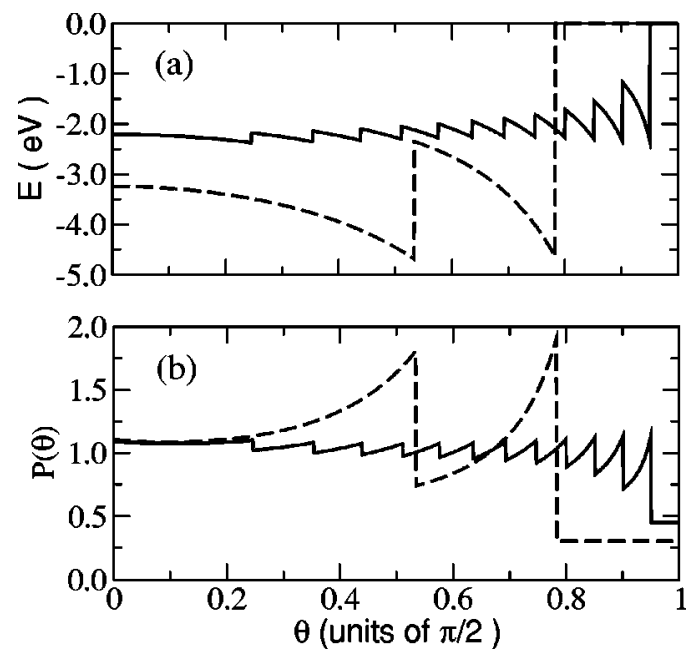

FIG. 2. (a) Total energy as a function of the coiling angle $\theta$ for polyacetylene (solid line) and PmPV (dashed line) wrapped around a $1 \mathrm{~nm}$ long nanotube of $1.3 \mathrm{~nm}$ diameter. (b) Corresponding probability density. The parameters for polyacetyle and PmPV are $\sigma=378 \mathrm{meV} / \mathrm{nm}^{2} ; \omega$ $=0.48 \mathrm{~nm} ; \quad D=32.5 \mathrm{meVnm}$ and $\sigma=770 \mathrm{meV} / \mathrm{nm}^{2} ; \quad \omega=2.1 \mathrm{~nm} ; \quad D$ $=250 \mathrm{meVnm}$, respectively.

be $m=\operatorname{INT}(2 \pi R \cos \theta / \omega)$, where $\operatorname{INT}(x)$ stands for the integer part of the argument $x$. We further assume that the polymer tends to minimize the total energy by adsorbing as many strands as possible to the walls of the nanotube. We thus define the dimensionless angle-dependent coverage as $\beta(\theta)=m \omega /(2 \pi R \cos \theta)$, which corresponds to the proportion of the unit-cell surface that is maximally covered by the polymer strands of width $\omega$. In terms of the coverage $\beta$, the total energy per unit length of nanotube can be written as

$$
E_{c}(\theta)=\frac{2 \pi R \beta(\theta)}{\omega} E_{1}(\theta) .
$$

As a function of the angle $\theta$, the coverage intermittently reaches $100 \%$ whenever the unit-cell surface area is an integer multiple of the surface area of one polymer strand. This full-coverage configuration is then followed by a discontinuous change in $\beta(\theta)$, meaning that one less polymer strand fits onto the wall of the nanotube. It should be pointed out that for an amorphous coating, the coverage never reaches $100 \%$ resulting in a higher minimum energy than in the crystalline case. Whereas the size of the discontinuities on the coverage is unimportant for narrow polymers, it is relevant for wide strands. In this case, the discontinuities on the coverage bring significant changes to the angle-dependent energy of the system. This is illustrated in Fig. 2(a), which shows the total energy $E$ as a function of $\theta$ for a $1.3 \mathrm{~nm}$ diameter tube of length $1 \mathrm{~nm}$ coated by PA (solid line) and PmPV (dashed line), two polymers with very different values of $\omega$. With narrow strands $(\omega=0.48 \mathrm{~nm})$, the former induces small variations on the coverage and, consequently, on the total energy. On the other hand, for the wide PmPV ( $\omega$ $=2.1 \mathrm{~nm}$ ), the fluctuations are much larger producing two distinct energy minima as a function of the angle $\theta$. By considering the Boltzmann factor $\exp \left(-E(\theta) / k_{b} T\right)$ associated with the energy curves of Fig. 2(a), we also plot in Fig. 2(b) the corresponding probability density $P(\theta)$ for different angles. Except for small variations, $P(\theta)$ is almost flat for $\mathrm{PA}$ and does not provide any clear preferential coating to AIP license or copyright; see http://apl.aip.org/apl/copyright.jsp 
angles. However, for PmPV, the two energy minima of Fig. 2(a) give rise to two respective peaks in the probability indicating preferential alignment directions at $\theta=48^{\circ}$ and $\theta$ $=70^{\circ}$ when this polymer coats the walls of a $1.3 \mathrm{~nm}$ diameter nanotube. This can be compared with the observation by scanning tunneling microscopy of a PmPV strand coiling around a $1.3 \mathrm{~nm}$ diameter SWNT with an angle of approximately $50^{\circ}$, in good agreement with the first peak. ${ }^{6}$ Such a good agreement with so simple a model is indicative that the geometric constraint does play a fundamental role in determining the preferential angles for crystalline polymers coating the wall of nanotubes. Furthermore, this model gives us predictive power to tailor the maximum-probability angle. By increasing the tube diameter, the probability curve shifts to the right-hand side up to the point where an additional peak appears at $\theta=0$. In this way, one can position the peaks of the probability function by adequately selecting the diameter range of the nanotube templates. Since the mechanical reinforcement of composite fibers is expected to correlate with the crystallization angles, this model is potentially capable of predicting the right combination of polymer/ nanotube to control the strength of these materials.

In summary, we have presented a simple model that describes the ordered assembly of polymer monolayers on nanotubes. Energetically favorable coiling angles result from geometric constraints that limit the maximum coverage of polymers on the wall of the nanotubes. More importantly, it is suggested that the angle can be adjusted by selecting the adequate range of nanotube diameters, opening the possibility of engineering the strength of composite fibers.

${ }^{1}$ S. J. Tans, A. R. M. Verschueren, and C. Dekker, Nature (London) 393, 49 (1998).

${ }^{2}$ R. H. Baughman, C. X. Cui, A. A. Zakhidov, Z. Iqbal, J. N. Barisci, G. M. Spinks, G. G. Wallace, A. Mazzoldi, D. De Rossi, A. G. Rinzler, O. Jaschinski, S. Roth, and M. Kertesz, Science 284, 1340 (1999).

${ }^{3}$ M. Cadek, J. N. Coleman, V. Barron, K. Hedicke, and W. J. Blau, Appl. Phys. Lett. 81, 5123 (2002).

${ }^{4}$ A. B. Dalton, S. Collins, E. Muoz, J. M. Razal, V. H. Ebron, J. P. Ferraris, J. N. Coleman, B. G. Kim, and R. H. Baughman, Nature (London) 423, 703 (2003).

${ }^{5}$ J. N Coleman, W. J. Blau, A. B. Dalton, E. Muoz, S. Collins, B. G. Kim, J. Razal, M. Selvidge, G. Vieiro, and R. H. Baughman, Appl. Phys. Lett. 82, 1682 (2003).

${ }^{6}$ B. McCarthy, J. N. Coleman, R. Czerw, A. B. Dalton, M. in het Panhuis, A. Maiti, A. Drury, P. Bernier, J. Nagy, B. Lahr, H. J. Byrne, D. L. Carroll, and W. J. Blau, J. Phys. Chem. B 106, 3087 (2002).

${ }^{7}$ R. Czerw, Z. X. Guo, P. M. Ajayan, Y. P. Sun, and D. L. Carrol, Nano Lett. 1, 423 (2001).

${ }^{8}$ V. Enkelmann, M. Monkenbusch, and G. Wegner, Polymer 23, 1553 (1982).

${ }^{9}$ M. in het Panhuis, A. Maiti, A. B. Dalton, A. van den Noort, J. N. Coleman, B. McCarthy, and W. J. Blau, J. Phys. Chem. B 109, 478 (2003).

${ }^{10}$ G. C. McIntosh, D. Tomanek, and Y. W. Park, Phys. Rev. B 67, 125419 (2003).

${ }^{11}$ E. Frey, K. Kroy, J. Wilhelm, and E. Sackmann in Dynamical networks in physics and biology, edited by D. Beysens and G. Forgacs (EDP Sciences, France, 1998).

${ }^{12}$ M. Severin and O. Inganas, Europhys. Lett. 25, 347 (1994).

${ }^{13}$ C. L. Gettinger, A. J. Heeger, J. M. Drake, and D. J. Pine, J. Chem. Phys. 101, 1673 (1994). 